Supporting Information 


\section{Partially Fluorinated Graphene: Structural and Electrical Characterization}

Lanxia Cheng ${ }^{1 *}$, Srikar Jandhyala ${ }^{2}$, Greg. Mordi ${ }^{3}$, Antonio T. Lucero ${ }^{I}$, Jie. Huang ${ }^{I}$, Angelica. Azcatl $^{l}$, Rafik Addou ${ }^{l}$, Robert M. Wallace ${ }^{1}$, Luigi Colombo ${ }^{4}$, Jiyoung Kim ${ }^{1 *}$

${ }^{1}$ Department of Materials Science and Engineering, The University of Texas at Dallas, 800 West Campbell Road, Richardson, TX 75080, USA

2 Currently at Intel Corporation, Hillsboro, OR 97124, USA

3 Currently at Samsung Austin Semiconductor, Austin, TX 78754

${ }^{4}$ Texas Instruments, Dallas, TX 75243, USA

* Corresponding authors: Tel:+1 972-883-6412, E-mail: jiyoung.kim@utdallas.edu, lanxia.cheng@utdallas.edu
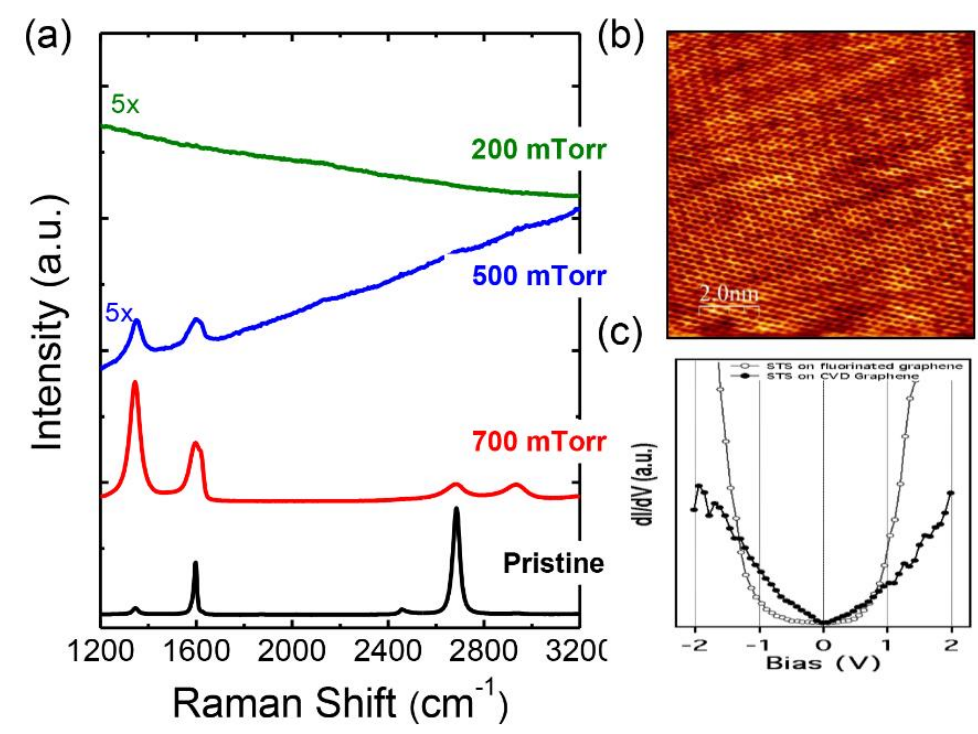

Figure S1. (a) Raman spectra showing the effect of $\mathrm{CF}_{4}$ partial pressure on the transferred CVD graphene on $\mathrm{SiO}_{2} / \mathrm{n}^{+} \mathrm{Si}$ substrate using plasma power of $40 \mathrm{~W}$ for $10 \mathrm{~s}$. For same period of plasma exposure time, higher pressure shows slower reaction between $\mathrm{CF}_{\mathrm{x}}$ radicals and carbon atoms in graphene, which allows a more controllable fluorination process. (b) STS of CVD graphene before and after fluorination showing the presence of band gap in fluorinated graphene. (c) STM image 
of the lattice structure of CVD graphene before fluorination recorded at $0.1 \mathrm{~V}, 0.7 \mathrm{nA}(10 \times 10$ $\mathrm{nm})$
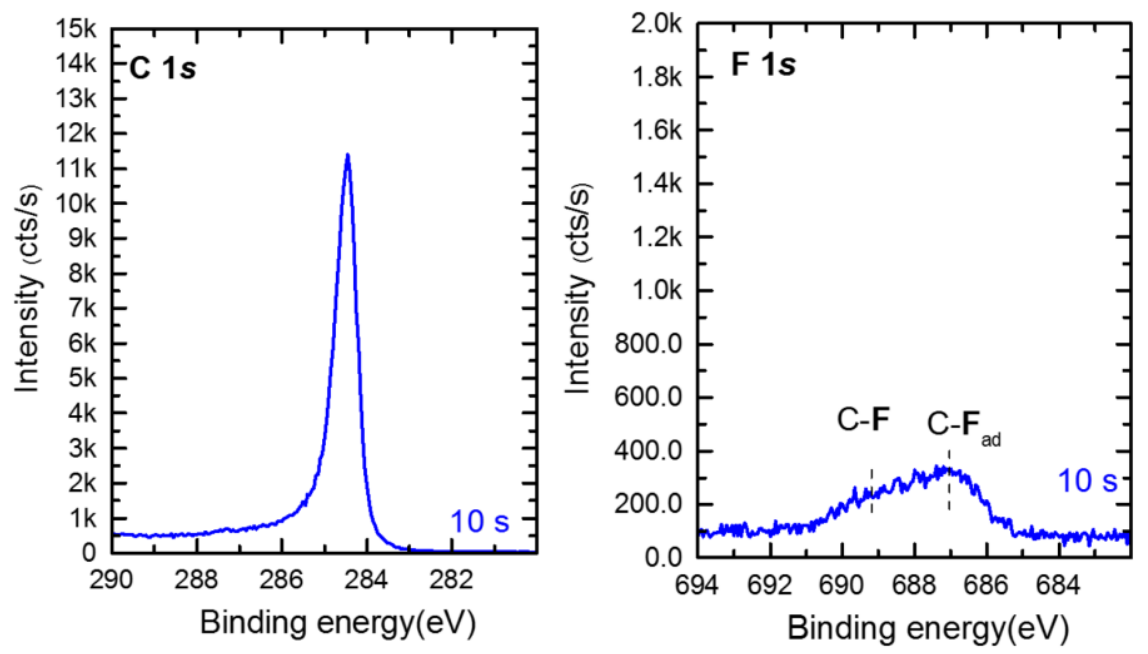

Figure S2. XPS of C 1s and F 1s binding states after $10 \mathrm{~s}$ fluorination on $\mathrm{HOPG}$ using $\mathrm{CF}_{4}$ plasma at $40 \mathrm{~W}$ and $700 \mathrm{mTorr}$.
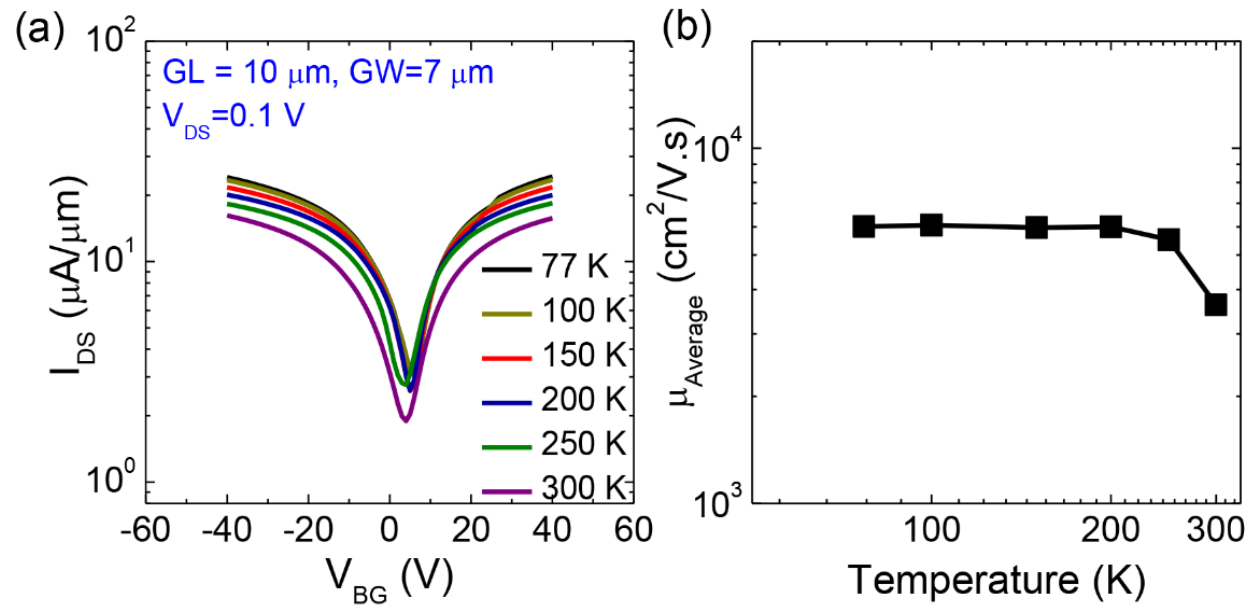

Figure S3. $\mathrm{I}_{D S}-\mathrm{V}_{\mathrm{BG}}$ curves and mobility of back-gated graphene devices as a function of temperature for exfoliated graphene. The channel dimension is $10 \times 7(\mathrm{~L} \times \mathrm{W}) \mathrm{um}$. All data was collected at $\mathrm{V}_{\mathrm{DS}}=0.1 \mathrm{~V}$ and it has been normalized with respect to the width of the graphene channel. 

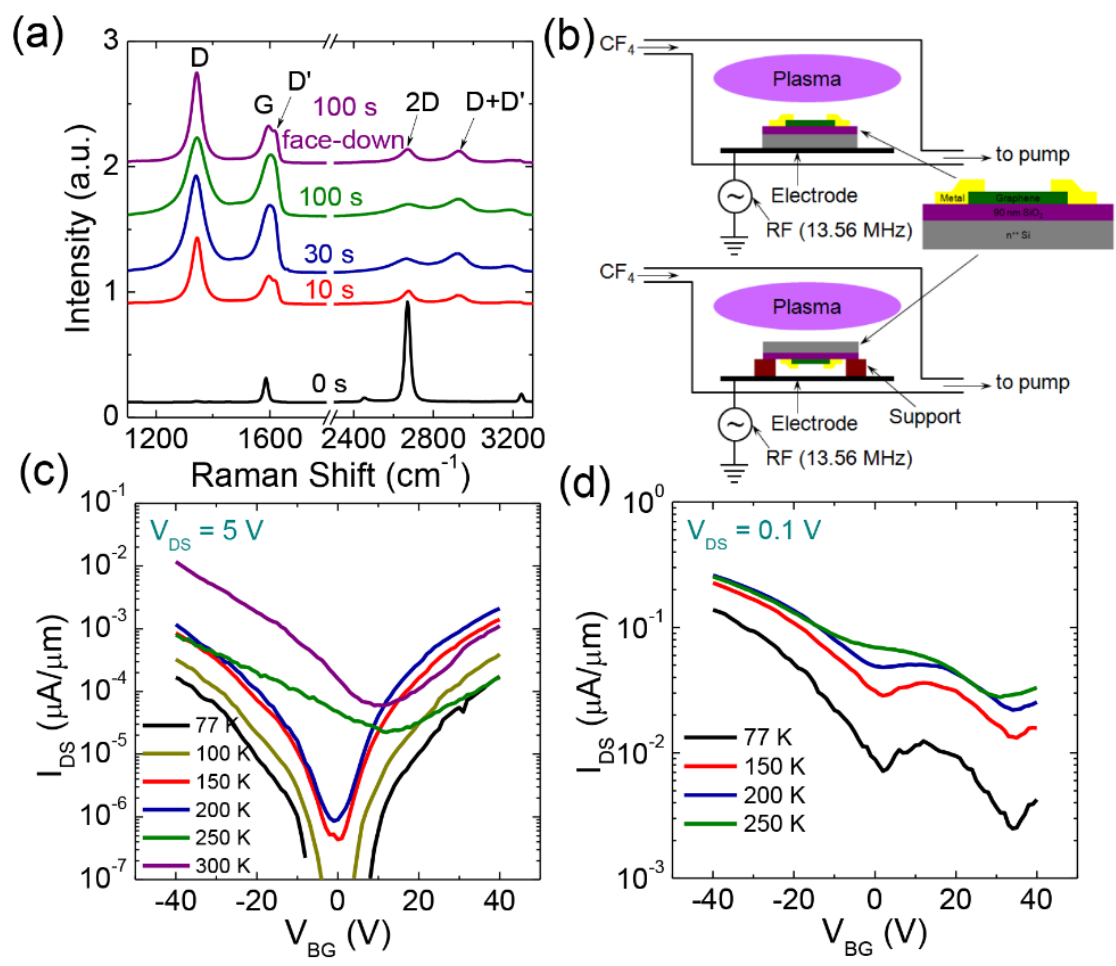

Figure S4. (a) Raman spectra of graphene after different fluorination times in facing down setup. (b) Schematics showing the setup for two different fluorination procedures: face-up (top) and facedown (bottom). (c)-(d) Transport properties ( $\mathrm{I}_{\mathrm{DS}}-\mathrm{V}_{\mathrm{BG}}$ curves) as a function of temperature of backgated (exfoliated) graphene FETs fluorinated for $100 \mathrm{~s}$ using (a) face-up and (b) face-down configurations. The channel dimensions of the back-gate GFETs shown in this data are: Length = $\sim 5 \mu \mathrm{m}$ (a) and $\sim 10 \mu \mathrm{m}$ (b), Width $=\sim 4 \mu \mathrm{m}$ (a) and $7 \mu \mathrm{m}$ (b). In the above figures, data was collected at $\mathrm{V}_{\mathrm{DS}}=5 \mathrm{~V}$ (c) or $0.1 \mathrm{~V}$ (d) and $\mathrm{I}_{\mathrm{DS}}$ has been normalized with respect to the width of the graphene channel. 
(a)

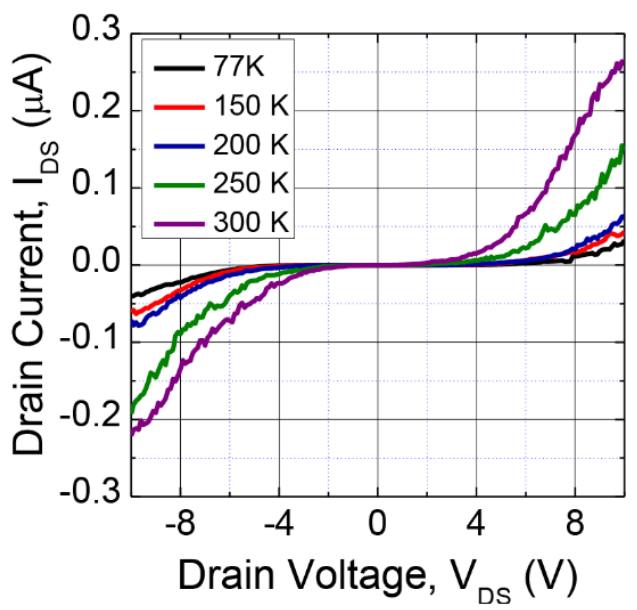

(b)

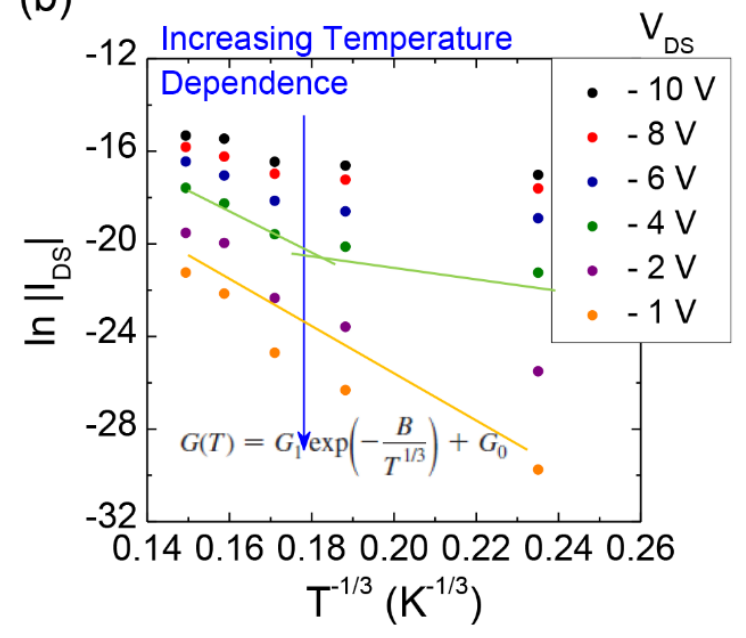

Figure S5. (a) Temperature dependent $\mathrm{IDS}_{D S} \mathrm{~V}_{D S}$ curves of a back-gated graphene FET after fluorination for $10 \mathrm{~s}$ at $700 \mathrm{mTorr}$ and $40 \mathrm{~W}$. All data was collected at $\mathrm{V}_{\mathrm{BG}}=0 \mathrm{~V}$. (b) Conductivity as a function of $\mathrm{T}^{-1 / 3}$ for different applied drain biases.
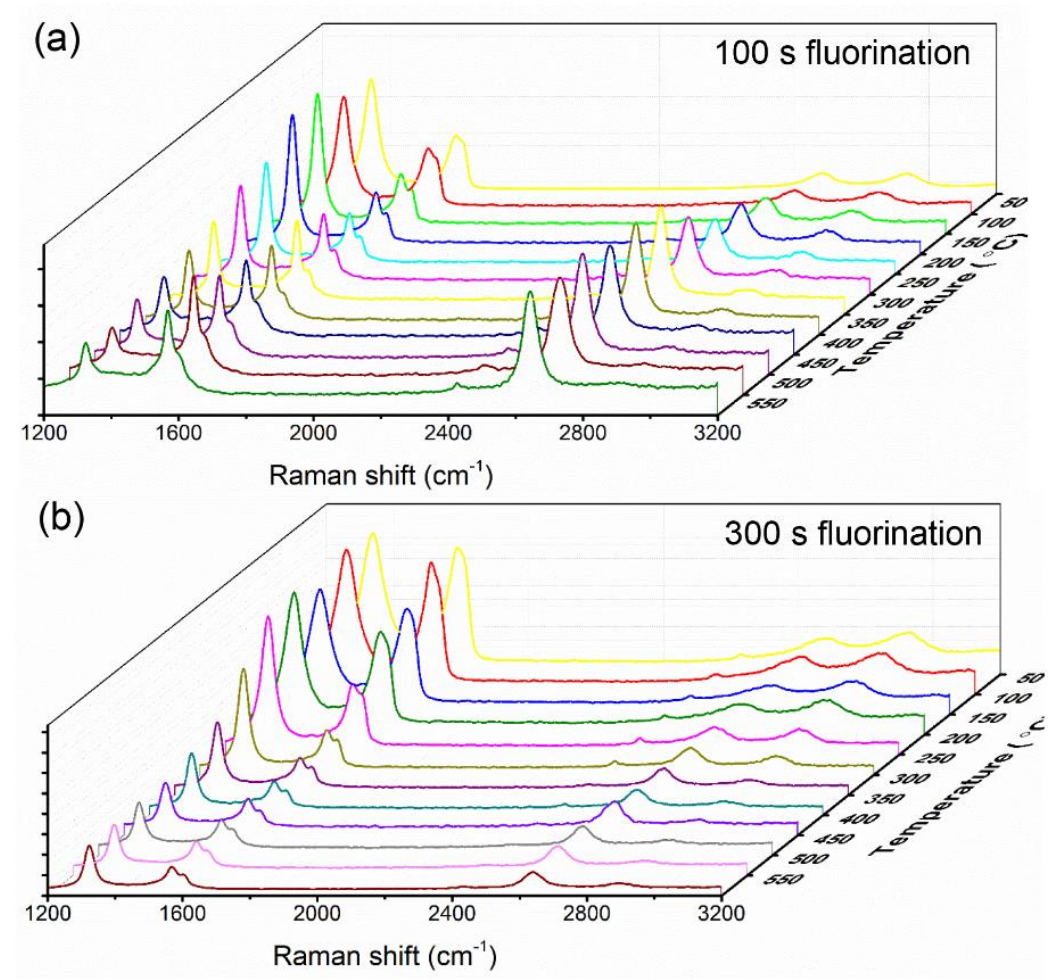

Figure S6. $(a, b)$ are the temperature dependent Raman spectra of CVD fluorinated graphene sheets produced from 100 and 300 s fluorinating at 700 mTorr and $40 \mathrm{~W}$ using $\mathrm{CF}_{4}$, respectively, 
showing evolution of Raman graphene features as function of annealing temperature collected at temperature interval of $50{ }^{\circ} \mathrm{C}$.

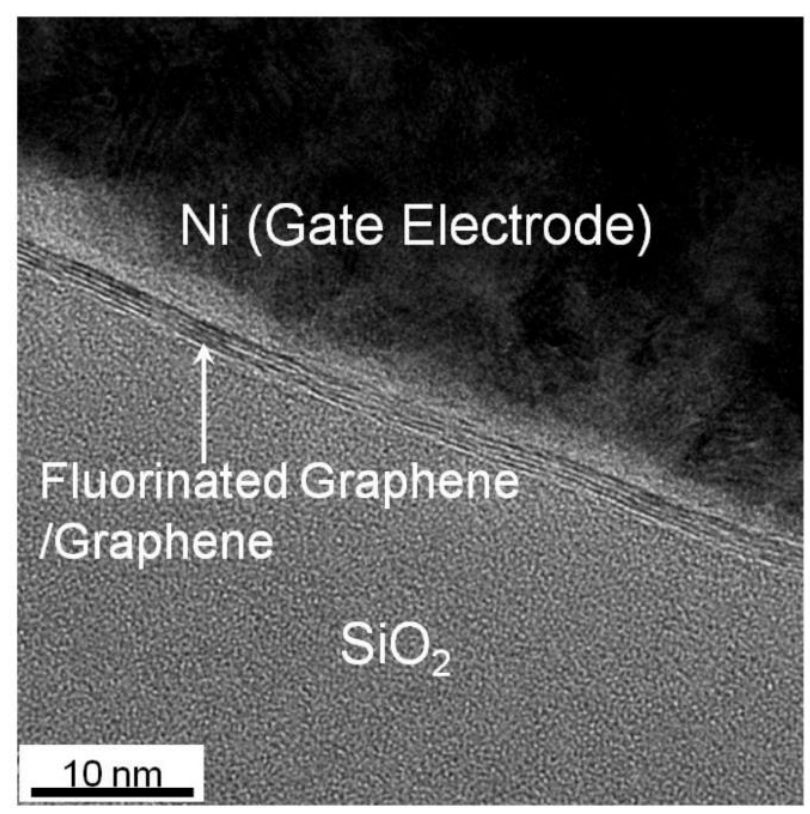

Figure S7. TEM cross-section images of fluorinated graphene (4 layers) stacked vertically on $\mathrm{SiO}_{2} / \mathrm{n}^{+} \mathrm{Si}$, suggesting a single layer fluorinated graphene thickness of $\sim 0.5 \mathrm{~nm}$. 